\title{
Neuroelectromagnetic Forward Modeling Toolbox
}

\author{
Zeynep Akalin Acar and Scott Makeig
}

\begin{abstract}
This paper introduces a Neuroelectromagnetic Forward Modeling Toolbox running under MATLAB (The Mathworks, Inc.) for generating realistic head models from available data (MRI and/or electrode locations) and for solving the forward problem of electro-magnetic source imaging numerically. The toolbox includes tools for segmenting scalp, skull, cerebrospinal fluid (CSF) and brain tissues from T1weighted magnetic resonance (MR) images. After extracting the segmented tissue volumes, mesh generation can be performed using deformable models. When MR images are not available, it is possible to warp a template head model to measured electrode locations to obtain a better-fitting realistic model. The Boundary Element Method (BEM) is used for the numerical solution of the forward problem. Toolbox functions can be called from either a graphic user interface or from the command line. Function help messages and a tutorial are included. The toolbox is freely available under the GNU Public License for noncommercial use and open source development.
\end{abstract}

\section{INTRODUCTION}

In brain electromagnetic source imaging (EMSI), the forward problem (FP) is to calculate the electromagnetic fields observed on or near to the scalp given a source distribution in the brain. For accurate source localization, the FP must be solved numerically using a realistic head model. This study introduces the Neuroelectromagnetic Forward Modeling (NFM) Toolbox, written in $\mathrm{C}++$ and MATLAB, that can be run from within EEGLAB (http://www.sccn.ucsd.edu/eeglab/) or used as a standalone solver. The NFM Toolbox uses METU-FP Toolkit (http://www.eee.metu.edu.tr/metu-fp/) as the realistic FP solver that uses the Boundary Element Method (BEM) and contains tools to generate BEM models from available subject data.

Source localization and source imaging are valuable tools for investigating electrical activity in the brain. The accuracy of source localization depends largely on the head model used for source localization. Realistic head models employing the Boundary Element Method (BEM) or the Finite Element Method (FEM) allow more accurate calculation of the electrical and magnetic fields compared with spherical head models. Furthermore, high quality and high performance BEM and FEM solvers are available to the scientific community ([1], [2], [3]). However, most researchers use either spherical head models or numerical models with relatively simple, template-based head models. The main reason for this is the difficulty of creating high quality, single subject,

This work is supported by Swartz Foundation, NY.

Z. Akalin Acar and S. Makeig are with the Swartz Center for Computational Neuroscience, Institute for Neural Computation, University of California San Diego, La Jolla, USA. [zeynep, scott] @sccn.ucsd.edu realistic head models. The NFM toolbox aims to assist the user in generating subject-specific head models using any and all available information about the subject and recording montage, and to provide an easy-to use interface for using this model with FP solvers.

Releasing the NFM toolbox under an open source license allows researchers to contribute and improve upon the work for the benefit of the neuroscience community. By bringing together the advanced head modeling and forward problem solution methods and implementations under an easy to use toolbox, this work complements EEGLAB [4], an open source toolkit under active development at UCSD. These toolboxes, when combined, will form a freely available EEG/MEG source imaging solution.

The toolbox implements the major components of realistic head modeling and FP solution from available subject information:

1) Segmentation of T1-weighted MR images: The preferred method of generating a realistic head model is to use 3-D whole-head structural MR images of the subject. The toolbox can generate a segmentation of scalp, skull, CSF and brain tissues from T1-weighted images.

2) High quality BEM meshes: The accuracy of the BEM solution depends on the quality of the underlying mesh that represent tissue conductance change boundaries. The mesh must be topologically correct with no selfintersections. It should represent the surface using high quality elements while keeping the number of elements as small as possible. NFM toolbox can create highquality linear or quadratic surface BEM meshes from the head segmentation and from fine surface meshes of the tissue boundaries.

3) Warping of a template head model: When a 3-D whole-head structural MR image of the subject is not available, a more realistic head model can be generated by warping a template BEM mesh to the digitized electrode coordinates.

4) Registration of electrode positions with the BEM mesh: The digitized electrode locations and the BEM mesh must be aligned to compute accurate FP solutions and lead field matrices.

5) Accurate and high performance FP solution: The NFM toolbox uses the high performance BEM implementation from the open-source METU-FP Toolkit for bioelectromagnetic field solutions.

The toolbox has two major parts: Generation of realistic head models and the numerical solution of the forward 
problem. The head model is either generated from MR images (if available) by segmentation and mesh generation or by warping a template head model to digitizer locations. The Toolbox functionality is explained in the next section. The third section describes the toolbox components, together with some screenshots from the GUI. In the Results section, examples of head models will be shown and the accuracy and efficiency of the BEM will be estimated.

\section{Toolbox Functionality}

In this section, the forward problem solutions, segmentation, mesh generation, warping and registration of the electrode locations is explained.

\section{A. Boundary Element Method}

The boundary element method (BEM) is a numerical computational technique for solving partial differential equations. In electro-magnetic source imaging (EMSI) of brain activity, it is used to solve the forward problem using realistic head models. When using BEM for head modeling, the head is assumed to be composed of uniform conductivity regions (i.e., scalp, skull, brain, etc.) and the tissue boundaries are represented by triangular surface elements.

The properties of the BEM implementation are summarized as follows:

1) To eliminate the singularity of the BEM matrix, the method of matrix deflation is employed.

2) An Isolated Problem Approach (IPA) implementation is extended to overcome numerical errors caused by high conductivity differences near the skull layer when there are multiple layers inside the skull [5].

3) A recursive integration technique is employed to increase the accuracy of the BEM implementation. In the recursive integration, the surface elements are divided into sub-elements and the numerical integration is performed on each sub-element. This process is repeated recursively until a subdivision criterion is met. Since the potential field is calculated at the original nodes, the size of the BEM matrix equation remains the same, but the accuracy of the surface integral is improved.

4) The BEM implementation allows the use of quadratic surface elements in realistic head models and is capable of handling intersecting surfaces.

5) To decrease the computation time, transfer matrices are computed that relate the sources to the fields at the sensor locations. Thus, by pre-computing the transfer matrices, forward solutions for electric and magnetic fields are reduced to simple matrix-vector multiplications.

\section{B. Segmentation}

When tomographic images of the subject's head are available, head tissue modeling is possible. Using only T1weighted MR images, a four-layer head model consisting of scalp, skull, CSF, and brain is generated. The CSF (inner skull) boundary is obtained using an eroded skull boundary and a dilated brain boundary. The segmentation algorithm is given in Figure 1.

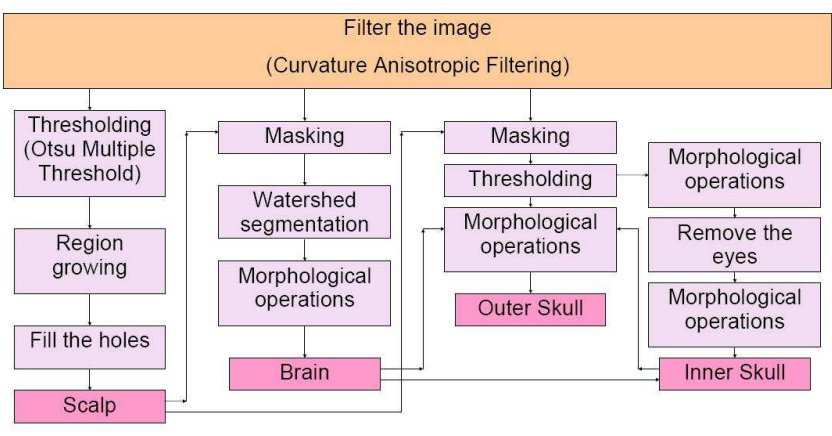

Fig. 1. Segmentation algorithm

\section{Mesh Generation}

To solve the forward problem, the geometrical information obtained by segmentation should be converted into a numerical form (i.e. a mesh). To generate the BEM surface meshes, an initial mesh is deformed iteratively until it matches the boundary of the segmented volume [6].

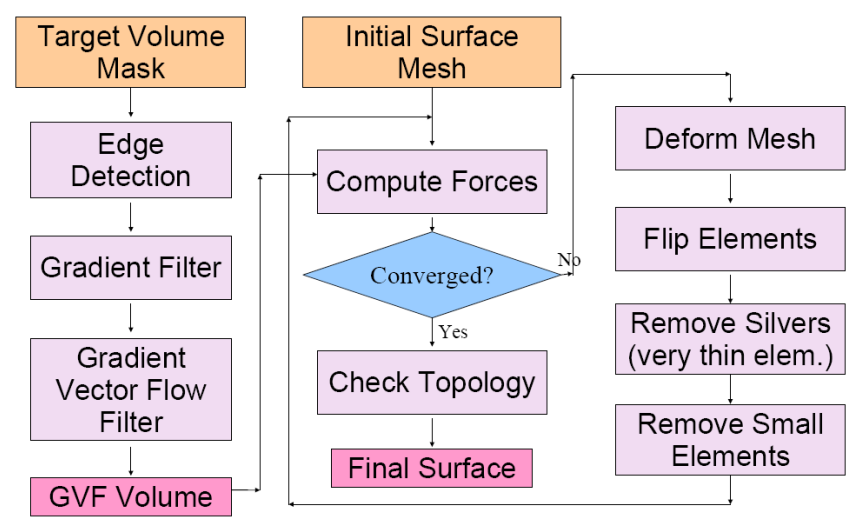

Fig. 2. Mesh generation algorithm.

\section{Warping}

For some subjects, tomographic head images may not be available; for their data, the only available spatial information may be the digitized locations of the EEG electrodes. For these subjects, a standardized head model is warped to the measured electrode locations ([7], [8]).

\section{E. Registration of electrode locations and scalp surface}

For registering the electrodes to the head model, a rigidbody transform is used. Six parameters for rotation and translation are determined that minimizes the distance between the BEM mesh and the electrode positions using a non-linear optimization algorithm. [1].

\section{TOOLBox COMPonents AND RESUlts}

In this section, the toolbox components are introduced. Input and output of each module is explanied with the screenshots of their user interfaces. 


\section{A. Head Modeling: Segmentation}

The inputs of the segmentation module are T1-weighted MR images and the outputs are filtered MR images and scalp, skull, CSF and brain masks. When the images are loaded, they are shown in sagittal, axial, and coronal directions and it is possible to move between slices easily. A volume selector chooses the image between MR images, filtered images or masks that will be displayed in the figures.

Segmentation steps are performed one by one:

1) Anisotropic filtering. (User inputs: filter parameters)

2) Scalp segmentation.

3) Brain segmentation. (User inputs: a seed for brain segmentation, lower end of the brain.)

4) Outer skull segmentation. (User inputs: seeds for eye lobes.)

5) Inner skull segmentation.

It is possible to save the results during any stage of segmentation in Matlab data format. The filtered image is saved seperately and the maskes are saved in a structure form. A snapshot from the segmentation user interface is shown in Figure 3.

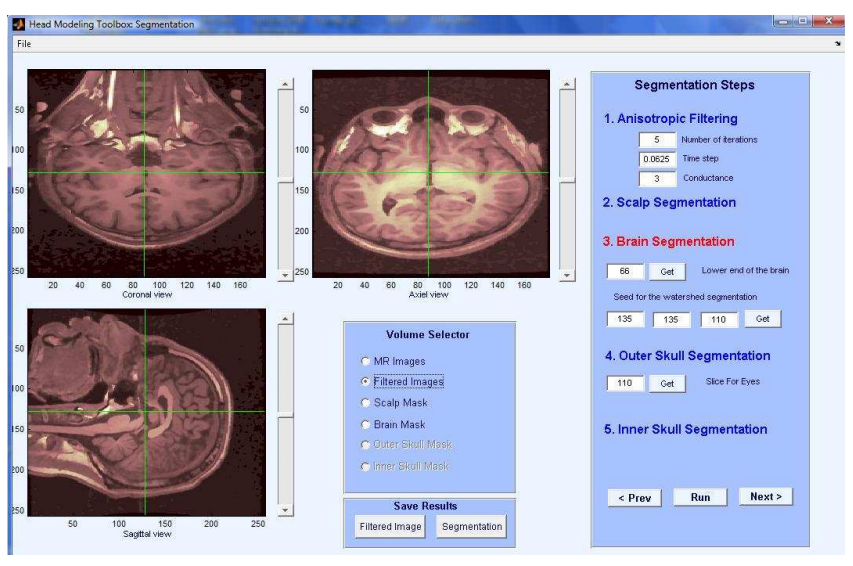

Fig. 3. Segmentation interface

\section{B. Head Modeling: Mesh Generation}

The Mesh Generation module is a stand alone program written in $\mathrm{C}++$. The module takes a segmented image and an initial mesh as its inputs, and outputs the surface mesh. The input mesh is deformed to match the volume. A snapshot from the mesh generation user interface is shown in Figure 4. In this study, a head model generated from 176-slice T1weighted sagital MR images with 1-mm slice thickness. In Figure 5, the meshes generated from the segmentation of the MR images are shown.

\section{Head Modeling: Warping}

The users selects between a fine and a coarse MNI template models for warping. The fine mesh has 15000 nodes, and the coarse mesh has 3000 nodes on each of the scalp, skull and CSF surfaces. The warping is computed based on fiducials: the nasion and left and right preauricular points. The digitized points are transformed onto the template

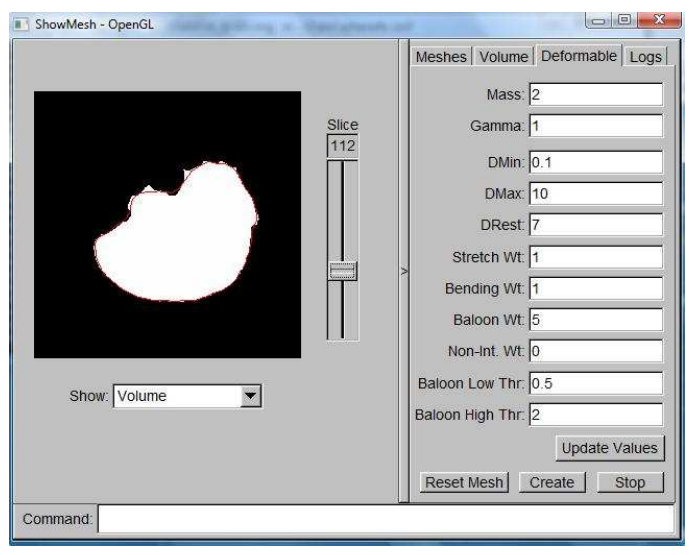

Fig. 4. Mesh generation interface

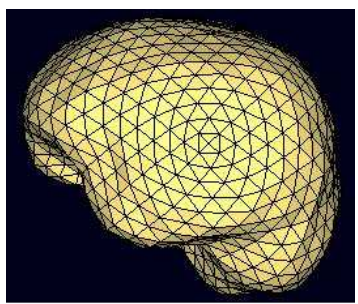

(a)

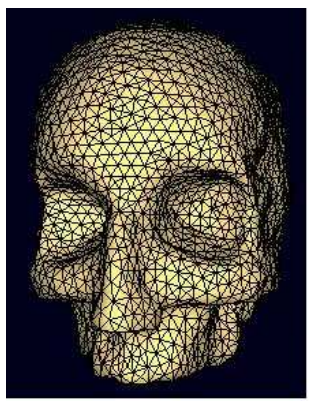

(c)

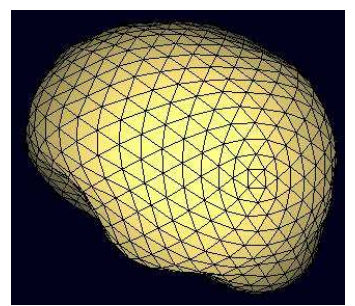

(b)

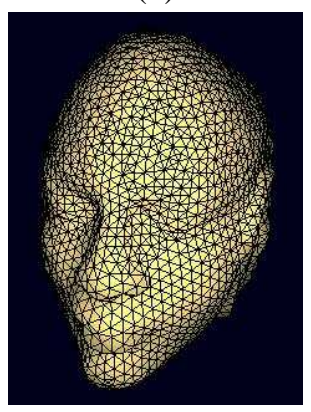

(d)
Fig. 5. BEM model of the scalp, skull, csf and the brain. (a) brain mesh, (b) CSF mesh, (c) skull mesh, (d) scalp mesh.

mesh and matching landmarks are located. The input of the warping module are the fiducials and the electrode locations (obtained from a digitizer). The outputs are the warped mesh, indices of the electrodes on the mesh (some of the electrodes may fall out of the mesh), fitted electrode locations and the warping parameters in case a user wants to warp back the localized sources to the template model. In Figure 6 a result of warping is shown in different views.

\section{Forward Problem Solutions: BEM}

The forward problem solution module is written mainly in MATLAB and has a set of functions to read the mesh, generate the BEM matrices, register the electrodes with the BEM mesh, and compute FP solutions and lead field matrices. These functions can also be accessed using the GUI. A snapshot from the BEM user interface is shown in Figure 7.

The MATLAB interface of the FP module uses three structures to store the state of FP computations: 

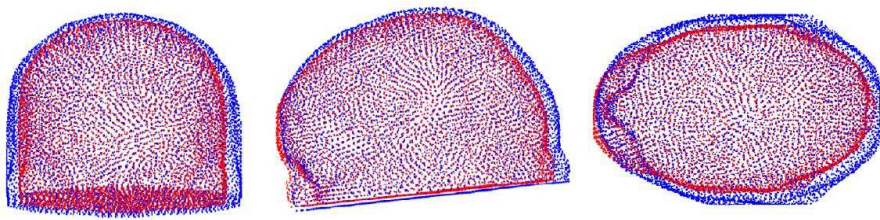

Fig. 6. The nodes of the template mesh (blue) and the warped mesh (red) are illustrated for different views. The warped mesh fits the digitizer locations.

- Mesh structure: stores the mesh information, ie., the geometry of the head.

- Model structure: contains the mesh and solver parameters (ie. IPA) and tissue conductivities.

- Montage structure: contains model and electrode coordinates on the mesh.

BEM solution steps:

- BEM matrices are generated for a specific head model and conductivity distribution and are stored on disk for future use.

- Transfer matrices are calculated for each experiment when the sensor locations are changed.

- The Lead Field Matrix or dipole source potentials are calculated using the transfer matrix for each source distribution.

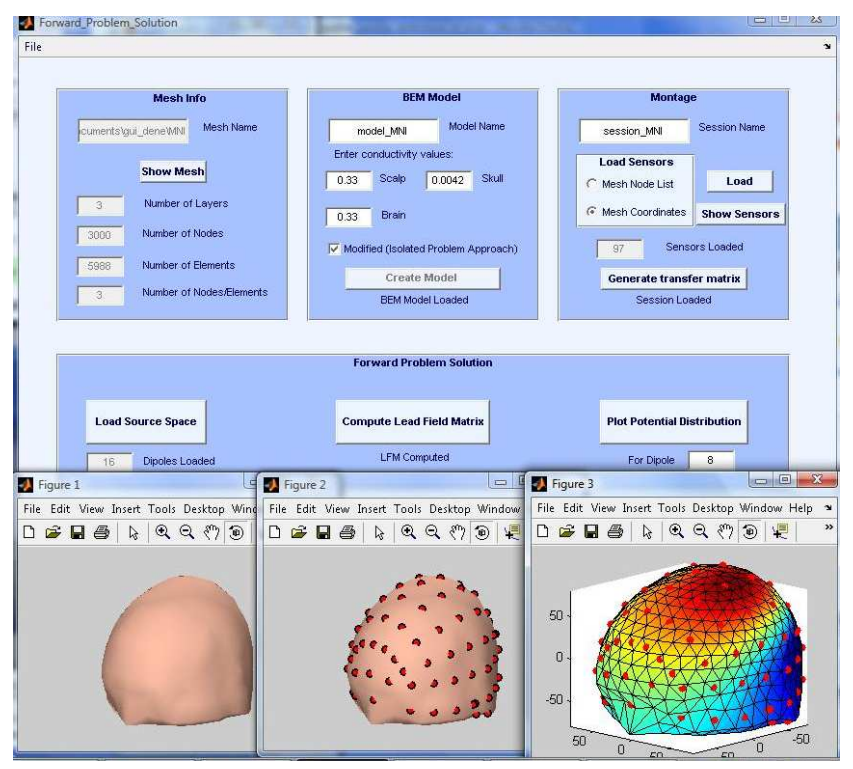

Fig. 7. BEM interface with figures

\section{CONCLUSIONS AND FUTURE WORKS}

\section{A. Conclusions}

Here, we introduce the Neuroelectromagnetic Forward Modeling toolbox, a collection of modules for generating and solving the EEG Forward Problem using realistic head models. Generation of realistic head models is accomplished using T1-weighted MR images or by warping a template mesh to digitizer locations. The Boundary Element Method is used to solve the forward problem. There is an interface to EEGLAB, and EEGLAB functions and data structures will be compatible with NHM Toolbox. The toolbox is now in alpha test stage; a beta version is expected to be released soon under the GPLv2 license.

\section{B. Future Works}

In future releases, we plan to add analytical solvers for concentric 3-4 layer spherical models. Numerical and analytical magnetic field solutions will be added. The segmentation will have an option to use multi-modal MR images, which will increase accuracy of CSF segmentation.

\section{ACKNOWLEDGMENTS}

The authors gratefully acknowledge Jeng-Ren Duann and Ruey-Song Huang for acquiring MR images.

\section{REFERENCES}

[1] Z. Akalin Acar, N.G. Gencer, An advanced BEM implementation for the forward problem of Electro-magnetic source imaging, Physics in Med. and Biol., vol. 49(5), 2004, pp 5011-28.

[2] N. G. Gencer, C. E. Acar, Sensitivity of EEG and MEG measurements to tissue conductivity, Phys. Med. Biol., vol. 49, 2004, pp 701-717.

[3] C.H. Wolters, M. Kuhn, A. Anwander, S. Reitzinger, A parallel algebraic multigrid solver for finite element method based source localization in the human brain, Comput. Visual Sci., vol. 5, 2002, pp 165-177.

[4] A. Delorme, S. Makeig, EEGLAB: an open source toolbox for analysis of single-trial EEG dynamics including independent component analysis, $J$ of Neuroscience M., vol. 134, 2004, pp 9-21.

[5] N.G. Gencer, Z. Akalin Acar, Use of the isolated problem approach for multi-compartment BEM models of electro-magnetic source imaging, Physics in Med. and Biol., vol. 50, 2005, pp 3007-22.

[6] JY. Park, T. McInerney, D. Terzopoulos, M. Kim, A non-selfintersecting adaptive deformable surface for complex boundary extraction from volumetric images, Computers and Graphics, vol. 25, 2001, pp 421-440.

[7] F. Darvas, J.J. Ermer, J.C. Mosher, R.M. Leahy, Generic head models for atlas-based EEG source analysis, Human Brain Mapping, vol. 27(2), 2005, pp 129-143.

[8] Z. Akalin Acar, S. Makeig, 'Neuroelectromagnetic Head Modeling Toolbox and EEGLAB Module', Soc. for Neuroscience, San Diego, 2007. 